\title{
Genomics: the next psychiatric revolution?
}

\author{
ANNE FARMER and MICHAEL J. OWEN
}

\begin{abstract}
"Genome" is a comparatively recent term that refers collectively to all the genetic material of an organism and "genomics" is an even newer word used to describe the study of genomes. The Human Genome Project is a coordinated effort of research taking place in the US, Europe, Japan and in many other countries that is aimed at characterising the human genome. It can be broadly conceived of in three parts. The first aim is to produce a detailed genetic map consisting of many thousands of ordered genetic markers which should in principle allow genes underlying any human inherited disease or trait to be located. The second stage is to provide a continuous physical map of DNA which will consist of sets of overlapping DNA clones, allowing the genes localised by genetic mapping to be identified. The third phase is to identify all the genes in the human genome, and to determine their DNA sequence and location. It is perhaps surprising to learn, given the breathtaking audacity of this endeavour, that the first two phases at least are proceeding well ahead of schedule. The current challenge is to develop more rapid and accurate methods of DNA sequencing but we can now envisage a time, certainly in the first half of the next century, when virtually all human genes will have been identified and sequenced.
\end{abstract}

\section{The Human Genome Project}

The Human Genome Project is the enabling technology by which the genes contributing to the genetic aetiology of common familial disorders, including the major psychiatric disorders, will be identified. We may be uncertain precisely how quickly and by what means such discoveries will be made (Owen \& McGuffin, 1992) but there is little doubt that they will happen and that the knowledge gained will radically alter clinical practice. Moreover, insights from genomics are unlikely to be restricted to disease. It is likely that we will gain insights into the genetic basis of behavioural traits such as personality, cognitive ability and sexual orientation. The complex issues raised and their implications for psychiatric practice need to be considered now. The accuracy of diagnosis of major psychiatric disorders will be greatly enhanced and the complex interplay between environment and genotype will be increasingly understood. Ultimately we can expect that the drugs produced as a result of understanding molecular pathogenesis will improve management and prognosis. However, the ability to predict which individuals are at risk of developing psychiatric disorder will raise ethical dilemmas. Understanding the genetic basis of human behaviour is also likely to bring benefits as it improves our understanding of mental handicap, neurosis and personality disorder. However, there is also concern that such information could be misused.

\section{Concerns about the potential misuse of psychiatric genotics}

It is not difficult to trace the historical routes of this disquiet. The first of these probably stems from a reaction to the eugenics movement in the early part of the century. Eugenics was based upon the optimistic notion that a knowledge of genetics could not only enable the abolition of certain diseases (negative eugenics) but could also lead to improvements of human stock in general (positive eugenics) (Carlson, 1987; Roll-Hanssen, 1988). Some believed that this could be implemented on a national basis, and misunderstandings about genetic and racial influences on IQ and other behavioural characteristics played a part in the way immigration policies were implemented in the USA (Kamin, 1974). However, it is well known there was much worse to come. Eugenic arguments were commandeered by the German Third Reich. It is particularly important to remember that despite the fact that such policies were seen by much of the scientific community at large as not just morally repugnant but intellectually flawed, they could not have been implemented in Germany to such a disastrous extent without the cooperation of large numbers of the medical establishment, including many psychiatrists (Proctor, 1988).

Another potent source of antipathy to psychiatric genetics is the view that genetic explanations of 
psychological disorders are unacceptably mechanistic and demeaningly simplistic, often allegedly ignoring the role of environmental factors such as poverty, child abuse, poor education and interpersonal relationships. In other words, discovery of a genetic contribution to a disease or trait is often mistakenly equated with genetic determinism. This argument is often allied with another which claims that not only do genetic theories reduce the richness and complexity of human experience but also that they offer only the prospect of therapeutic nihilism because genetic mechanisms must be immutable and are certainly unlikely to be susceptible to psychological or social interventions. A related concern is that interest in genetics will lead to the neglect of potentially remediable non-biological contributions to mental illness (Pelosi \& David, 1989).

However, counter-arguments to these are not difficult to find. Identifying the genes underlying susceptibility to major psychiatric disorders will inevitably lead to an understanding of the relationship between genetic and environmental factors. Indeed, for many of the major psychiatric disorders, the environmental agents have proved elusive (McGuffin et al, 1994), and it is possible that most will only be identified as a result of identifying the genes with which they interact. There are a number of examples already of genetic diseases where environmental manipulations form the basis of treatment such as phenylketonuria and there is no reason why finding that there are genetic influences on a disorder should rule out psychological or behavioural treatments. For example, twin studies suggest that obsessive-compulsive disorder is heritable (MoGuffin et al, 1994) but the disorder is responsive to cognitive-behavioural psychotherapy. Other well known examples include the effectiveness of cognitive therapy in major depression and the usual combination of drug plus family therapy in prolonging remissions in schizophrenia. However, clearly both the profession and the public need information and reassurance on these points. It would also be naive to suppose that increased understanding of the genetics of psychiatric and behavioural disorders will not lead to specific ethical problems. We shall now consider how these might relate directly to clinical practice.

\section{Ethical problems arising from the availability of predictive testing in Huntington's disease (HD)}

Well before neuropathological mechanisms can be completely understood it will be possible to identify those individuals with a genetic propensity to develop psychiatric disorder. Such predictive testing is already available for Huntington's disease (HD) and some of the ethical issues posed by the availability of such testing have been considered (Morris \& Harper, 1991; McGuffin et al, 1994).

Huntington's disease was the first neuropsychiatric disorder where predictive tests became available. Autosomal dominant transmission, close to $100 \%$ penetrance and the recent identification of the causal mutation (Huntington's Disease Collaborative Research Group, 1993) ensure that individuals at risk for $\mathrm{HD}$ can be confidently assigned to either very high or very low risk categories. Two types of predictive test are now available. The first is based on direct mutational analysis, while the second type is pre-natal exclusion testing of a child of an at-risk parent.

The ethical concerns that have been considered regarding such tests are as follows: Some individuals have been inappropriately referred for testing while others were not informed of the referral by the referrer. Both instances indicate the need for better information and education about predictive testing in HD. Expert counselling before, and psychological support after, testing if the outcome is adverse have been recommended. Unintentional risk alteration remains a problem even now direct mutational analysis is possible (e.g. a parent being shown to have the mutation by the testing of their offspring). However, this can be avoided in prenatal exclusions where what is at issue is whether the unborn child shares the $50 \%$ risk of the parent with a family history of the disorder. Such issues also indicate the need for pre-test counselling and this recommendation forms part of the agreed guidelines for predictive testing in HD. Also agreed was the inappropriateness of testing in children despite requests to do so from parents and others such as adoption agencies.

Another issue which has arisen out of the availability of predictive testing in HD relates to who has the right to know the test results. Experience has shown that the majority of those who request the test do not proceed after initial counselling. Clearly the "right not to know" must be protected. Employers and insurance companies may also consider that they should be informed of test results. Indeed it is possible to foresee the situation where life insurance cover will not be available without a genetic profile being undertaken to determine individual risks for all common diseases, including psychiatric disorder. Whether this can be considered as acceptable or not requires 
wider public debate, it is clearly not just a matter for the profession. These issues are likely to be even more problematic in societies in which medical care is largely private and based upon health insurance.

As we have already discussed prenatal diagnosis of high risk HD offspring can also be made. In addition pre-natal testing can already determine the presence of foetal abnormalities such as neural tube defects or Down's syndrome. Decisions regarding termination must be made with the fullest information and with careful counselling of the couple concerned. Societal pressure for termination should not be placed on such couples merely on the grounds of cost of caring for a disabled individual.

Guidelines that are appropriate for predictive testing in HD may not necessarily pertain to other psychiatric disorders where the modes of transmission are often more complex. Implications of recent genetic discoveries in Alzheimer's disease (AD) allow the two situations to be contrasted and are instructive.

\section{Recent genetic discoveries in Alzheimer's disease (AD)}

Initial molecular genetic research focused upon autosomal dominant early onset ( $<65$ years) familial Alzheimer's disease. Mutations in the gene in coding the beta amyloid precursor protein on the long arm of chromosome 21 are found in approximately $10 \%$ of such families (McGuffin et al, 1994). However, the majority of families have mutations in a recently discovered gene on the long arm of chromosome 14 (Sherrington et al, 1995). In addition, there is a third group of such families in which disease is caused by mutation on chromosome 1 (Rogaev et al, 1995). In these pedigrees with clear autosomal dominant transmission the clinical consequences of genetic research are similar to those in Huntington's disease and clinicians should seek involvement from the genetic counselling services if DNA testing is contemplated.

However, clear autosomal dominant transmission is seen in less than $1 \%$ of all cases of $A D$. The genetics of common, late onset forms of the disorder appear more complex, and it seems likely that the majority of cases result from the coaction or interaction of several, perhaps many, different genes. One of these seems to be the Apolipoprotein E (ApoE) gene on chromosome 19. Many studies have now shown an association between both early and late onset sporadic Alzheimer's disease with the 84 allele of the ApoE gene. The frequency of 84 is 0.35 to 0.52 in patients compared with approxi- mately 0.15 in controls. However, some $40 \%$ of people with $A D$ do not possess 84 , so this allele is neither necessary nor sufficient to cause the disease and other aetiological factors and other genes must play a part. If a polygenic multifactorial mode of transmission is assumed then 84 accounts for about $17 \%$ of the total variance in liability and less than half of the genetic variance (Owen et al, 1994).

The predictive value of ApoE genotyping in young people is low and systematic screening of presymptomatic individuals will be of little value (Owen et al, 1994, Nalbantoglu et al, 1994). The usefulness of ApoE genotyping alone as a diagnostic test remains to be determined, though the initial indicators are not good as there is some evidence that $\varepsilon 4$ may be increased in other forms of dementia. This is currently an area of intensive research activity and there is hope that it may be possible to use ApoE genotyping to identify subgroups of patients in terms of therapeutic response or disease progression.

\section{Presymptomatic testing in other psychiatric disorders}

The genetics of other psychiatric disorders such as schizophrenia and bipolar disorder are also complex and again several, perhaps many genes are involved. However, current large scale genotyping will probably reveal loci for these disorders before the end of the decade. Unlike AD, which usually commences after decades of normal life, the peak incidence for schizophrenia and bipolar disorder is during the late teens and early twenties. Ethical issues relating to pre-symptomatic predictive testing are likely to be different again from those pertaining to testing for $\mathrm{HD}$ and autosomal dominant $\mathrm{AD}$. Onset of the disorders in early adult life raises issues about the desirability of testing in adolescence. Clearly predictive testing would only be of value in advance of emerging psychopathology. Unlike both HD and AD however, advice can be given to individuals with high genetic loading for these disorders regarding exposure to environmental precipitants such as the use of street drugs. In addition drug treatments for both schizophrenia and bipolar disorder are well established and highly effective. Thus presymptomatic testing may offer greater individual benefits than are currently the case for $\mathrm{HD}$ and $\mathrm{AD}$ where no effective treatment is available. Indeed, presymptomatic identification of high risk individuals may also lead to earlier diagnosis and treatment when symptoms do emerge. 


\section{Conclusions}

Predictive testing for the main disorders will provide the start of the genomic revolution in psychiatry. However, other developments will follow which will inevitably have a major impact on clinical practice. The ability to correct the effects of faulty genes could lead to prevention or cure. The knowledge base will be radically altered and psychiatry may become more allied to mainstream medicine, since the pathogenic mechanisms underlying psychiatric disorders like those of other common diseases such as coronary artery disease and hypertension will be understood at the molecular level. Enhanced knowledge of the genetics of abnormal behavioural traits will almost cerainly provide effective means for altering such behaviour. It is clear that complex moral and ethical issues will be raised.

History reminds us of the past human rights abuses carried out in the name of eugenics. Thus, it is imperative that careful, informed consideration of such issues are made now so that safeguards can be put in place. Poorly informed legislation remains a risk even in the last decade of the 20th century (Bobrow, 1995). By contrast legally imposed limits on artificial fertilisation and human embryo research show that sensible and humane policies can be made which are acceptable to the majority of both the public and professions.

There are clearly many more ethical and moral issues that will arise when the explosion of genetic knowledge occurs in psychiatry. It is better for the profession to approach these with foresight rather than be overtaken by the pace of events.
References
Bonkow, M. (1995) Redrafted Chinese law remains eugenic. Journal of Medical Genetics, 32, 409.

CArLson, E. A. (1987) Eugenics and basic genetics. History and Phillasophy of Life Sciences, 3, 57-78.

Huntmatons Desense Collaborative Resenarch Group (1993) A novel gene containing a trinucleotide repeat that is expanded and unatable on Huntington's disease chromosomes. Cell, 72, 1-20. Kumm, L. J. (1974) The Science and Politics of IQ. Chichester. Wiley.

McGupfin, P., Owen, M. J., O'Donovan, M., el al (1994) Seminars in Psychiatric Genetics. London: Gaskell.

MonRs, M. J. \& HARrer, P. S. (1991) Prodiction and prevention in Huntington's disease. In: The New Genetics and Mental Illhess (ods P. MoGuffin \& R. Murray), pp. 281-298. Oxford: Butterworth-Heinemann.

NAliantoolu, J., Gifix, B. M. Bertrand, P. et al (1994) Predictive value of Apolipoprotein E genotyping in Alzheimer's discase: results of an autopsy series and an analysis of several combined studies. Annals of Neurology, 36, 889-895.

OWEN, M. J. \& MCGUPFIN, P. (1992) The molecular penetics of echizophrenia. (Editoria). British Medical Journal, 305, 664-665. , LIDDEL, M. B. \& MCGUFFIN, P. (1994) Alzheimer's disease. An asecciation with apolipoprotein $\mathrm{E} A$ may help unlock the puzade. (Editorial). British Medical Journal, 308, 672-673.

PEwoe, A. \& DAVID, A. (1989) Ethical implication of the new genetic for poychiatry. International Review of Psychiatry, 1 315-320.

Proctor, R. N. (1988) Racial Hygiene Medicine under the Nazis. Cambridge MASS: Harvard University Press.

Roul-Hunssen, N. (1988) The progreas of eugenics: growth of knowlodge and change in ideology. History of Science, 26, 295-331.

Rogaev, E. I., Sherrennoton, R., Rogaeva, E. A., el al (1995) Familial Alzheimer's disease in kindreds with missense mutations in one on chromosome 1 related to the Alzheimer's disease type 3 gene. Nature, 376, 775-779.

Shisarnioton, R., Rogaev, E. I., LuNo, Y., et al (1995) Cloning of a ene bearing misense mutations in early onset familia Alzheimer's disease. Nature, 375, 754-760.

Anne Farmer, FRCPsych, Department of Psychological Medicine; Michael J. Owen, MRCPsych, Department of Medical Genetics, University of Wales College of Medicine

Correspondence: Professor Anne Farmer, Department of Psychological Medicine, University of Wales College of Medicine, Heath Park, Cardiff CF4 4XN

(First received 28 September 1995, final revision 13 February 1996, accepted 20 February 1996) 